\title{
Iron status of children with attention deficit/hyperactivity disorder: A systematic review
}

\author{
Alexia Degremont ${ }^{1}$, Rishika Jain ${ }^{1}$, Elena Philippou ${ }^{1,2}$ and Gladys Oluyemisi Latunde-Dada ${ }^{1}$ \\ ${ }^{1}$ Department of Nutrition and Dietetics, King's College London, London, United Kingdom and \\ ${ }^{2}$ Department of Life and Health Sciences, University of Nicosia, Nicosia, Cyprus
}

Important advances have been made in understanding the pathophysiology of attention deficit/hyperactivity disorder (ADHD) ${ }^{(1)}$. While focus was on examining systemic iron status ${ }^{(2,3)}$, brain iron status may be a more valuable biomarker of the disorder ${ }^{(4)}$. The close relationship of iron and dopaminergic transmission in the brain, which was found diminished in ADHD, as well as the uncertain correlation of serum iron and brain iron concentrations ${ }^{(5)}$, support the requirement for direct measurement of brain iron status. With the above in mind, the present systematic review aimed to examine whether children with ADHD have lower serum as well as brain iron content, compared to healthy controls.

A systematic literature search in Medline via PubMed, the Cochrane Library, Web of Science, Embase and Ovid for papers between 2000 to June $7^{\text {th }} 2019$, was conducted using the following terms; ADHD or attention deficit hyperactivity disorder or hyperactivity and iron status or iron deficiency or iron deficient anaemia or anaemia or ferritin or brain iron. Studies were included if one of the outcomes was the mean difference of iron concentration, measured as serum iron, serum ferritin or brain iron, between children with ADHD and healthy controls. Studies performed on adults, animal studies, narrative and systematic reviews, meta-analyses, abstracts and reports, as well as studies published not in English were excluded. The systematic review followed Preferred Reporting Items for Systematic Reviews and Meta-Analyses (PRISMA) guidelines. Risks of bias within and between studies were assessed using the quality assessment tools of the National Heart, Lung and Blood Institute of the National Institutes of Health (NIH, 2014) for Case-Control Studies and for Observational Cohort and Cross-Sectional Studies.

Out of 599 records screened, twenty case-control studies met the inclusion criteria. 10/18 studies found a significant difference in serum ferritin and 2/10 studies observed a significant difference in serum iron between children with ADHD and healthy controls. Overall, results on systemic iron concentration are inconsistent. The three studies that assessed brain iron consistently found a statistically significantly lower thalamic iron concentration in children with ADHD compared to healthy controls. Meta-analysis was not performed because three different outcomes, using different assays, were investigated.

Limited evidence supported that brain iron level is a better biomarker of ADHD than serum iron level in children. Larger longitudinal magnetic resonance imaging studies are needed in order to examine the correlation of iron deficiency in specific brain regions and symptoms of ADHD. Effects of iron supplementation on these symptoms at various time points should also be further investigated.

1. Hare D, Ayton S, Bush A et al. (2013) Front Aging Neurosci 5, 34.

2. Tseng P, Cheng Y, Yen C et al. (2018) Sci Rep 8(1), 788.

3. Wang Y, Huang L, Zhang L et al. (2017) PloS One 12(1).

4. Adisetiyo V, Helpern J (2015) Biomark Med 9(5), 403-406.

5. Cortese S, Azoulay R, Castellanos F, et al. (2012) World J Biol Psychia 13(3), 233-231. 